\section{Single-cell immune landscape of human recurrent}

$$
\text { spontaneous abortion }
$$

Feiyang Wang ${ }^{1,4, \#}$, Wentong Jia ${ }^{1,4, \#}$, Mengjie Fan²,\#, Zhilang Li ${ }^{1,4}$, Yongjie $\mathrm{Liu}^{3,4}$, Yeling Ma ${ }^{1,4}$, Xuan Shao ${ }^{1,4}$, Yu-xia $\mathrm{Li}^{1}$, Rong $\mathrm{Li}^{2,{ }^{*}}$, Qiang Tu${ }^{3,4,{ }^{*}}$, Yan-Ling Wang $^{1,4,5,{ }^{*}}$

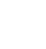

1. State Key Laboratory of Stem Cell and Reproductive Biology, Institute of Zoology, Chinese Academy of Sciences, Beijing 100101, China

2. National Clinical Center for Obstetrics and Gynecology, Peking University Third Hospital, Beijing 100191, China

3. State Key Laboratory of Molecular Developmental Biology, Institute of

Genetics and Developmental Biology, Innovation Academy for Seed Design,

Chinese Academy of Sciences, Beijing 100101, China

4. University of Chinese Academy of Sciences, Beijing 101408, China

5. Lead contact

\#, These authors contribute equally to this work

8 *, All correspondence should be addressed to Dr. Yan-Ling Wang at wangyl@ioz.ac.cn, or Dr. Qiang Tu at qtu@genetics.ac.cn, or Dr. Rong Li at roseli001@sina.com 


\section{SUMMARY}

Successful pregnancy in placental mammals substantially depends on the establishment of maternal immune tolerance to the semi-allogenic fetus. Disorders in this process are tightly associated with adverse pregnancy outcomes including recurrent spontaneous abortion (RSA). However, an in-depth understanding of the disorders from the aspect of systematic and decidual immune environment in RSA remains largely lacking. In this study, we utilized single-cell RNA-sequencing to comparably analyze the cellular and molecular signatures of decidual and peripheral leukocytes in normal and RSA pregnancies at the early stage of gestation. Integrative analysis identified 22 distinct cell clusters in total, and a dramatic difference in leukocyte subsets and molecular properties in RSA cases was revealed. Specifically, the cytotoxic properties of CD8T effector, NK, and MAIT cells in peripheral blood indicated apparently enhanced immune inflammatory status, and the subpopulation proportions and ligand-receptor interactions of the decidual leukocyte subsets demonstrated preferential immune activation in RSA patients. The molecular features, spatial distribution and the developmental trajectories of five decidual NK (dNK) subsets were illustrated. The proportion of a dNK subset responsible for fetal protection was reduced, while the ratio of another dNK subset with cytotoxic and immune-active signature was significantly increased. Notably, a unique pro-inflammatory CD56+CD16+ dNK subpopulation was substantially accumulated in RSA decidua. These findings reveal a comprehensive cellular 
45 and molecular atlas of decidual and peripheral leukocytes in human early

46 pregnancy, which provides an in-depth insight into the immune pathogenesis

47 for early pregnancy loss.

48

49 KEY WORDS: Decidual and peripheral leukocytes, single-cell RNA-seq, early

50 pregnancy, developmental trajectory

51

52 


\section{INTRODUCTION}

Reproductive success in placental mammals substantially depends on the establishment of maternal immune tolerance to the semi-allogenic fetus during pregnancy (Ander et al., 2019; Erlebacher, 2013). In humans, failures in immune tolerance are tightly associated with various adverse pregnancy outcomes, mainly including recurrent spontaneous abortion (RSA), preeclampsia, and stillbirth (Deshmukh and Way, 2019; Fisher, 2015; Kheshtchin et al., 2010)

The key of fine-tuned immune tolerance at the feto-maternal interface is the well-coordinated crosstalk among uterine mucosal immune cells and extraembryonic placental cells. The maternal immune cells populating the uterine mucosa include decidual NK (dNK) cells ( 50-70\%), macrophages ( 20\%), T cells $(\sim 10-20 \%)$ and a small amount of DC and B cells. The numerically dominant dNK cells are characterized by low cytotoxicity and strong cytokine producing capacity, which is divergent from peripheral NK (pNK) cells (Wang et al., 2014). Mechanistically, the inhibitory receptors LILRB2 and KIR2DL4 on dNK cells recognize HLA-G on the surface of trophoblast cells, leading to the suppressed cytotoxic capacity of dNK cells (Gamliel et al., 2018a; Parham and Moffett, 2013). Moreover, the biased differentiation of CD4+ T cells towards Th2 and Treg has been confirmed vital to the tolerance of fetal cells in decidua (Wang et al., 2010; Yang et al., 2008). 
75 characterized as multiple subsets that present dynamical lineage differentiation and crosstalk properties during pregnancy (PrabhuDas et al., 2015). However, the corresponding systematic study on human decidua is largely lacking, which hampers the in-depth understanding of the physio-pathological regulation of human pregnancy. A recent paradigm shifting study by Moffett and colleagues utilizing single-cell RNA-sequencing and comprehensive data analysis constructed a detailed cellular map and the elaborate cell-cell communication patterns of the human decidual-placental interface. Specifically, three major subsets of dNK cells that have distinctive immunomodulatory and chemokine profiles were identified (Huhn et al., 2020; Suryawanshi et al., 2018; Vento-Tormo et al., 2018). However, high-resolution immune landscape of dysregulated decidua in the contexts of pregnancy complications, for example in RSA, is still unknown, which greatly limit the exploration of immune-related pathogenesis for recurrent miscarriage. In the present study, we utilized single-cell RNA-sequencing to comparably analyze the cellular and molecular signatures of decidual and peripheral leukocytes in normal and RSA pregnancies at the early stage of gestation. By integrating the gene expression properties, ligand-receptor interactions, and spatial localization patterns, the cell-type-specific communications among various subsets of leukocyte and the developmental trajectory of dNK cells were constructed. A pathological leukocyte atlas in RSA decidua and peripheral blood was illustrated. Our findings reveal a detailed molecular and 
97 cellular map of decidual and peripheral leukocytes in early pregnancy of 98 human, and highlight the integral harmful immune responses that may lead to 99 early pregnancy failure.

\section{RESULTS}

\section{Clustering of immune cells from peripheral blood and decidual tissues in}

normal and RSA pregnancies

To characterize the immune cells in RSA, we applied scRNA-seq to study

CD45+ cells isolated from peripheral blood and decidual tissues of three pairs

of normal pregnant women and RSA patients with unknown cause. Viable

CD45+ leukocytes were enriched by FACS and subjected to droplet-based single-cell RNA sequencing (scRNA-seq) using the 10x Genomics Chromium and six decidual tissue samples were obtained from 12 scRNA-seq libraries.

111 Following computational quality control and filtering using the Seurat package

112 (Butler et al., 2018; Stuart et al., 2019), the final datasets containing 56,758

113 high-quality cells were subjected to further analysis (Fig. S1C, D and E).

We performed an unsupervised graph-based clustering to analyze scRNA-seq data in Seurat (version 3.0.3). In order to eliminate unreasonable

117 clustering due to batch effects, we integrated the data using the reciprocal PCA

118 function of Seurat. Overall, 22 transcriptionally distinct clusters were identified 
119 (Fig. 1A, B and C), and were further confirmed using the MNN algorithm 120 (Haghverdi et al., 2018)(Supplementary Fig. S2). The cell identity of the 22 121 clusters was assigned on the basis of known marker genes and literature 122 evidence (Vento-Tormo et al., 2018)(Suryawanshi et al., 2018) (Fig. 1D).

123 In peripheral blood, T cells were identified and grouped into five major clusters, 124 including two subsets of Naïve T cells (CD4T Naive and CD8T Naive) which 125 express the marker gene CCR7, mucosal-associated invariant T cells (MAIT) specifically expressing ZBTB16, CD4 Memory T cells (CD4T Memory) 127 annotated by S100A4, and CD8 T effector cells (CD8T effector) that express 128 marker genes FCGR3A and FGFBP2 (Fig. 1D; Supplementary FigS3). 129 Peripheral NK cells were clustered as CD56dimCD16+ NK cells (NK dim) and CD56brightCD16- NK cells (NK bright). Monocyte included CD14+ subset and 131 CD16+ subset. In addition, a low abundance of of B cells (identified by CD79A expression) and DC cells (annotated by $L Y Z$ and FCER1A) were captured in 133 peripheral blood.

In decidual tissues, the most abundant population appeared as dNK cells, which were subgrouped into five clusters, named as dNKa, dNKb, dNKc, dNkd, 137 and dNKe. The dNKe subset presented positive expression for FCGR3A/CD16, 138 which was uniquely accumulated in RSA decidual tissues. Decidual T cells 139 were clustered as CD4+, CD8+, and a relatively small number of FOXP3+ 140 regulatory $\mathrm{T}$ cells (dCD4T, dCD8T, and dTreg). Other decidual leukocyte 
141 clusters included KIT-expressing ILC3 cells (ILC3), CD14-expressing 142 macrophage (dM), FCER1A-expressing dendric cells (DC), and 143 TPSAB1-marked mast cells (Mast) (Fig. 1A and D).

Cellular and molecular characteristics of the peripheral leukocytes

147 Given $\mathrm{T}$ cells present a dominant proportion in peripheral leukocytes, we

148 therefore first analyzed the properties of $\mathrm{T}$ cell clusters in RSA and normal 149 pregnancies. The gene expression profiles of the five peripheral $\mathrm{T}$ cells 150 subsets were indistinguishable between RSA and normal pregnancies (Fig. $1512 \mathrm{~A}$ ). However, the proportion of each T cell cluster exhibited evident alterations 152 in RSA patients, presenting by reduced CD4 T Naïve, CD8 T Naïve and CD4 T 153 Memory populations, and augmented CD8 T effector and MAIT populations 154 (Fig. 2B). In addition, transcription of inflammatory factors, such as IRF1, 155 RORA in MAIT cells and IFNG in CD8 T effector cells and what was remarkably increased in RSA cases (Fig. 2C and D).

159 increased proportion of CD56dimCD16+ NK dim cells (Fig. 2E). Furthermore, 160 in these cells, evidently decreased expression of immunosuppressive genes, 161 such as TIGIT, KLRG1, IL32 and ALOX5AP, while increase in pro-inflammation genes including $H L A-B, N R 4 A 2, J U N, J U N B, N F K B I A$ and 
163

164

165

166

167

168

169

170

171

172

173

174

175

176

177

178

179

180

181

182

183

184

MAP3K8 were observed in RSA cases (Fig. 2F). Take together, the properties of the peripheral leukocytes, including aforementioned cell composition shift and specific gene expression pattern in certain cell cluster, strongly indicate an overall enhanced systematic pro-inflammatory immune feature of RSA patients.

\section{Cellular atlas of the decidual leukocytes in RSA pregnancy}

Increasing evidence has demonstrated compromised immune cell differentiation or function in RSA decidua. However, a global cellular change remains largely undefined. Our analysis of cell subset proportion in decidua clearly revealed an increased portion of dCD8 T, dNKd, and dNKe populations, and lowered ratio of dNKa, dNKc, and dM cells in RSA decidua (Fig. 3A). To systematically investigate the cell-cell communications among these cell subsets, we analyzed the expression levels of immune cell revenant ligand-receptor interacting pairs within cell types (Fig. 3B and C). Significantly increased expression of IFNG and TNF was found in dCD8 T, ILC3 and dM in RSA decidua, which may account for the accumulation of dNKe or dCD4T cells that exhibited remarkably higher expression of the corresponding receptors for these two cytokines. The receptor for CXCL16, i.e. CXCR6, was predominantly expressed in dCD4T and dCD8T. Therefore, the accumulation of T cell subsets in RSA decidua may attribute to the enhanced CXCL16 in dM. In addition, the expression of CCL3, CCL4, and CCL5 in different immune cell subsets also 
reflected the activation of the immune micro-environment at the maternal-fetal interface and the vigorous recruitment of macrophages (Du et al., 2014). Such molecular interactions among cell populations via specific ligand-receptor complexes indicated a potential mechanism of immune activation that caused compromised immune environment in RSA decidua.

\section{Differential gene expression profiles in decidual macrophage of RSA}

\section{pregnancy}

Alteration in global gene expression profiles in the RSA leukocyte subset was typically observed in $\mathrm{dM}$. As shown in the volcano plot, using fold-change (FC) $>1$ and $p$-values $<0.05$ as cutoffs, 96 upregulated genes and 27 downregulated genes were identified in RSA dM relative to its normal counterpart (Fig. 3D).

as antigen presentation, phagocytosis, recruitment of other immune cells (Varol et al., 2015)(Gentek et al., 2014). The increased expression of CXCL8, TNF, and IFIT2 genes in RSA dM indicated the impaired immune protection. Moreover, the suppressed level of angiogenic factor VEGFA and the immunosuppressive molecule LAGLAS1, while enhanced expression of pro-inflammatory factors CXCL8 and TNF in RSA dM cell were observed (Fig. $3 E)$. These data indicated a preferentially pro-inflammatory property of $d M$ in 
RSA patients.

208

Molecular aspects and developmental trajectories of human dNK subtypes

211 Consistent with the findings in previous literatures (Bulmer et al., 1991; King et 212 al., 1991), dNK cells accounted for more than $70 \%$ of total decidual leukocytes 213 in our dataset. The characteristics of the five dNK subpopulations were further 214 illustrated by the top 20 marker genes (Fig. 4A) and KEGG analysis (Fig. 4B). 215 As shown, dNKa cells highly expressed MCM5, STMN1, and PCNA, indicating 216 their manifest proliferative capacity. The defining markers for dNKb cells 217 included XCL1, XCL2, and ZNF683. dNKc cells strongly expressed ENTPD1 218 (CD39), CSF1 and PKM, and showed active oxidative phosphorylation and 219 glycolysis activity. dNKd cells were defined by ITGAE (CD103), CD160, and $220 K L R B 1$, and exhibited certain cytotoxic and immune-activated property. 221 FCGR3A/CD16, CXCR4, PLAC8 were enriched in dNKe cells, which indicates an active cytotoxicity status of these cells.

It has been well known that $\mathrm{dNK}$ cells possess various receptors for recognition of HLA-C, HLA-G and HLA-E molecules in placental trophoblast cells, which primarily mediates dNK-trophoblast communications. We found that dNKc cells expressed much high level of KIR2DL3 and KIR2DL1 which 
228

229

are inhibitory KIR receptor for HLA-C, and $L I L R B 1$, the high-affinity receptor for HLA-G. dNKc and dNKb cells also moderately expressed KLRC2/NKG2C, $K L R C 3 / \mathrm{NKG} 2 \mathrm{E}$ and $K L R C 1 / \mathrm{NKG} 2 \mathrm{~A}$, which are receptors for HLA-E. dNKd cells possessed much lower expression of all these receptors. Moderate levels of $L I L R B 1, K L R C 2 / N K G 2 C$ and $K L R C 3 / N K G 2 E$ were observed in dNKe cells (Fig. 4C).

To determine whether the dNK subtypes exhibit cell type dependent localization pattern at the feto-maternal interface, we performed immunofluorescent staining for CD56, ENTPD1(CD39), ITGAE(CD103), CD16 and PLAC8 on serial sections of decidual tissues. Immunohistochemistry for cytokeratin 7 (CK7) was used to locate trophoblast and uterine gland epithelium in the sections. The results showed that CD39 positive dNKc cells were more abundant in decidual compacta where trophoblasts infiltrate, while CD103-labelled dNKd cells mainly distributed in distal compacta through spongiosa, but less in proximal compacta (Fig. 5A, B and C). A very small number of CD16-expressing dNKe cells could be found in decidual compacta, usually near uterine spiral artery (Fig. 5D). These results predict the potential of $\mathrm{dNKc}$ cells in the recognition and protection of trophoblasts, and dNKd cells in maintaining certain cytotoxic and immune-active environment in decidua.

Interestingly, apart from dNKe, the other four dNK subtypes co-expressed the tissue-resident markers CD49a/ITGA1, indicating their uterine resident 
250

251

252

feature. By far the developmental route of uterine resident dNK cells remains unclear. Our result of flow cytometry showed dNKc cells as CD27-CD11b- (Fig. 4D), which indicates their immature developmental status according to the previous report (Fu et al., 2011). Therefore, we further analyzed the developmental trajectory of dNK cells by carrying out pseudotime analysis on our scRNA-seq data. The top 100 genes in each dNK subtype were clustered (Fig. S4), and Monocle2 was performed to infer their development trajectory (Fig. 4E). As shown, dNKa appeared at the very initiation of the trajectory, and dNKc emerge at the early developmental stage with much higher expression of dNK-classical transcription factors EOMES and ID3. It tended to differentiate towards dNKb and finally dNKd (Fig. 4F). Distance between dNKd and dNKe indicated the probably alternative origin of CD49a-CD16+ dNKe cells.

\section{Alterations of dNK subsets in decidua of RSA pregnancy}

To verify the feature of dNK subset proportion in RSA decidua identified by scRNA-seq in Fig 3A, we analyzed dNK cell population by flow cytometry in a larger sample cohort of normal and RSA decidual tissues. We sorted cells by using CD56 (expressed by all dNK cells) and CD3 (negative sorting marker), combined with markers for dNKc (CD39), dNKd (CD103), and dNKe (CD16) that were identified from our scRNA-seq data (Fig. 6A). As indeed, the cell portion of dNKc was uniformly and remarkably reduced by around $50 \%$, 
271 whereas dNKd proportion increased by 1-fold in RSA decidua, compared to

272 the normal pregnancy controls (Fig. 6B). The ratio of dNKe cells was rather

273 small in normal decidua, accounting for less than $4 \%$ of total dNK. However, in

274 approximately half of the enrolled RSA cases, dNKe cell population increased

275 to about $12 \%$ of total NK cells (Fig. 6B). Consistently, immunofluorescent

276 staining for CD16 in RSA decidua showed evidently more dNKe cells in

277 decidua compacta niche close to blood vessels (Fig. 5D).

RSA and normal pregnancy demonstrated a significant increased expression

281 of inflammation-related genes such as CD69 and IRF9 in dNKd cells, INFG,

282 CXCL8, TNFRSF8, and IFIT3 in dNKe cells, as well as downregulation of 283 anti-inflammation genes including SPINK2 and AREG in dNKc cells from RSA 284 patients (Fig. 6C). 


\section{DISCUSSION}

289

290

Cellular and molecular mechanisms accounting for maternal immune tolerance to protect growth of semi-allogenic fetus in placental mammals has been a fascinating scientific question. Increasing evidence demonstrate the significance of the complicated and precisely controlled cell-cell communications within the feto-maternal interface in concert with the well-coordinated systematic immune adaptations along gestation (Ander et al., 2019; Arck and Hecher, 2013; Erlebacher, 2013; Hanna et al., 2006; Nancy et al., 2012). Recently, a molecular and cellular map of normal feto-maternal interface at human early gestation strikingly deepened our understanding of the cell subtypes and their predicted interactions in normal homeostatic decidua (Vento-Tormo et al., 2018). However, the corresponding landscape and dynamics of immune cell at single cell level in failed pregnancy, such as RSA, is lacking to date. To our knowledge, our study is the first comprehensive single-cell transcriptomics atlas of the decidual and peripheral immune cells in human recurrent miscarriage at early gestation. Our findings integrate complex information of immune cell, including cell composition, functional status, developmental trajectory, and predicted cellular interactions among immune cells. Our work illustrates an integral framework of the compromised immune environment that is harmful to the intra-uterine fetus in RSA pregnancy. 
310 chromosomal or congenital abnormalities or other known pathological causes,

311 has been largely debatable. Multiple studies demonstrated abnormal

312 expressions or functions of various cytokines, or dysregulated proportions or

313 properties of certain immune cells in patients with recurrent miscarriage.

314 However, studies solely focusing on certain limited cell types may result in

315 misleading points or contradictive results. To date the strategies of early

316 diagnosis or intervention of RSA are lacking, which makes it hard to reduce the

317 threat to the patients (Seshadri and Sunkara, 2014). Here by taking

318 advantage of single-cell sequencing technology, we construct an overall

319 pathological change in the properties of immune cell subsets in peripheral

320 blood, which may pave the path for the pre-symptom diagnosis of the

321 disordered pregnancy. The significantly enhanced differentiation of naive $\mathrm{T}$

322 cells to CD8T effector cells, and preferential increasing in number of NK dim

323 cells, together with the upregulated inflammation-related genes, demonstrate a

324 systematic pro-inflammatory status in RSA patients. These results are

325 consistent with previous reports (Ebina et al., 2017; Kuon et al., 2017). An

326 interesting finding is the increased frequency of MAIT cells in RSA peripheral

327 blood. As a non-conventional T cell subset, MAIT cells are mainly activated by

328 exposure to microbes, while they can also be turned on by inflammatory stimuli

329 in the absence of TCR-mediated antigen recognition. There is considerable

330 evidence to suggest their involvement in a broad range of infectious and

331 non-infectious diseases (Godfrey et al., 2019). Studies in liver disorders 
suggest that the MAIT cells may play a protective role against bacterial infections in a normal liver, but might be detrimental, with over-inflammation, in liver diseases (Bertrand and Lehuen, 2019). Notably, MAIT cells express several cytokine receptors, including IL-1R, IL-7R, IL-12R, IL-15R, IL-18R and IL-23R, and response to these multiple cytokines stimulation (Godfrey et al., 2019). Our sequencing data showed the evidently enhanced expression of IRF1 and RORA in MAIT from RSA periphery, indicating their highly immune-activated property. By far evidence of MAIT cell in pregnant condition has been very limited, and our study for the first time indicate the association of MAIT activation with the maternal inflammation condition that damage fetal survival. Whether the change of MAIT activation is the cause or result of miscarriage remains to be established.

population of decidual leukocytes at early pregnancy. Unlike their peripheral counterparts, dNK cells are less cytotoxic but actively secret a vast array of factors and cytokines. They have been found to play powerful roles including facilitating the remodeling of uterine spiral arteries, promoting trophoblast invasion and fetal growth, regulating $\mathrm{T}$ cell differentiation, increasing the availability of maternal blood at the implantation site, and so on (Ander et al., 2019). In unexplained infertile patients, substantially fewer uterine NK cells were observed when compared to fertile controls (Klentzeris et al., 1994). 
However, discrepant results have been reported regarding the change of dNK cell number in RSA patients (King et al., 2010; Seshadri and Sunkara, 2014). Our study clearly demonstrates the abnormal properties of dNK subsets in RSA decidua, including cell composition and gene expression, which suggests impaired regulation of dNK subset development in the patients.

The characteristics of dNKc cells are similar to the highly active dNK1 subset in Vento-Tormo's study. They are numerically dominant dNK cells and represent the typical dNK functions to support embryo growth. The CD11b-CD27- property of dNKc manifests their immature characteristic and the potential to differentiate (Fu et al., 2011). Specific expression of PBX1 was found in CD27-CD11b- dNK cells, and a recent study demonstrated the association of decreased PBX1 expression or PBX1G21S mutation with unexplained RSA (Zhou et al., 2020), further suggesting the crucial role of this dNK subset in pregnancy maintenance. Interestingly, a recently identified pregnancy trained dNK (PTdNK) share similar features with dNKc or dNK1 (Gamliel et al., 2018b), and this subset has been speculated to be enriched and boost decidua receptivity in subsequent pregnancy, therefore may be responsible for the "memory" of reproductive outcomes in next pregnancies. Thus, the strikingly lowered frequency of this subset in RSA patients can partly explain why failed pregnancies repeatedly occur in the patients. In addition to the decreased proportion, the altered gene expression in dNKc of RSA decidua further indicates their diminished immune-protective capability. 
The development trajectory of dNK subsets reveals dNKd cells are relatively mature and immune-activated. They are likely to maintain certain cytotoxic and immune-active environment in decidua, which may contribute to the appropriate extent of trophoblast invasion. However, in RSA decidua their portion raises to more than one fold of the normal counterpart. Besides, they exert enhanced production of pro-inflammatory cytokines, which is likely causing the decidual environment harmful to the fetal cells and other maternal immune cells such as T cells and macrophages (Fridman et al., 2012; Fu et al., 2013; Ma et al., 2017; Sotnikova et al., 2014; Yang et al., 2019).

Another notable dNK subset in our study is the newly-identified CD56+CD16+ dNKe, which is largely enriched in RSA decidua. Being different from all the other four subsets, dNKe cells do not exert expression of the tissue-resident marker CD49a. Consistently, the developmental trajectory show separation of dNKe from the maturation route of other dNK subsets. It is therefore likely that this subset may origin from other sources. Currently there

393 are three hypotheses for the origin of dNK cells, i.e., 1) recruitment of 394 peripheral blood NK cells to decidua(Carlino et al., 2008), 2) maturation of 395 uterine-resident NK cells in response to IL15 or progesterone(Manaster et al., 396 2008), 3) direct differentiation from hematopoietic precursors in the decidua 397 upon stimulation of specific decidual factors(Vacca et al., 2011). Considering 
398

399

400

the common CD49a-CD16+ feature of dNKe and peripheral NK dim, we further compared the gene expression pattern between dNKe and NK dim. As shown in the volcano plot, using fold-change $(F C)>1$ and $p$-values $<0.05$ as cutoffs, 187 upregulated genes and 73 downregulated genes were identified in dNKe relative to NK dim. However, there are 699 co-expressed genes between dNKe and NK dim, which present their common feature in exercising common cellular functions (Fig. S5). Thus, it is likely that peripheral NK dim cells are recruited to decidua and further educated towards dNKe by some decidual factors. Many studies have demonstrated the crucial role of IFN-gama, CCL3/MIP-1, CXCL10/IP-10, CXCL12/SDF-1 in enrolling pNK to decidua (Hanna et al., 2003; Wallace et al., 2013; Wu et al., 2005). What's more, an in vitro study reveals that human peripheral CD16+ NK cells can be converted to a dNK-like phenotype upon the stimulation of hypoxia, TGF-beta, and the demethylating agent Aza (Zhang et al., 2016). Here our data reveals the evident increase in INFG in dCD8T, CXCL16, CCL3 and TGFB in dM of RSA decidua, which may be responsible for enrolling and educating excessive pNK cells to decidua compartment. The much higher expression of several pro-inflammatory factors in RSA dNKe cells also indicate their enhanced cytotoxicity in the patients. An interesting previous study demonstrated the extravagant enrichment of CD16+ NK cells in endometrium of RSA patients during their pre-pregnant period (Lachapelle et al., 1996). Therefore, we propose that the patients with more abundant dNKe cells may suffer a greater 
420

421

422

423

424

425

426

chance of pregnancy failure in their subsequent pregnancies.

Decidual macrophages account for around $20 \%$ of leukocytes at the feto-maternal interface, and they have many diverse functions during pregnancy. Here a relatively large amount of differentially expressed genes was identified in RSA decidual macrophages, which potentially suggest some functional abnormalities. For instance, the obviously repressed VEGFA in RSA $\mathrm{dM}$ indicates the link to impaired remodeling of the spiral arteries and angiogenesis. The upregulated genes include immunoinflammatory factors and the relevant signaling molecules, such as CXCL8, TNF, IFIT2, JUN, JUNB, etc., predicting the diminishment in the anti-inflammatory capacity. Furthermore, the increased expression of IL-8 in $\mathrm{dM}$ and enhanced IFNG expression in dNKe of RSA patients are likely in tight correlation, because it has been demonstrated that IL-8 from dM enhances the production of IFN-gama in dNK cells (Baratin et al., 2005; Dalbeth et al., 2004). In addition, our brief repository of ligand-receptor complexes indicated the potential of $\mathrm{dM}$ in recruiting dCD8T or dNKe through CXCL16-CXCR6 or TNF-TNFRSF1B interactions. Although decidual macrophages are believed to exist predominantly in a regulatory/homeostatic M2-like (dM2) phenotype, while less in pro-inflammatory M1 (dM1) phenotype during pregnancy(Erlebacher, 2013), we did not find an alteration in the proportion of $\mathrm{dM} 1$ and $\mathrm{dM} 2$ in RSA patients (Fig. S6). This is probably due to the relatively small amount of captured dM 
cells for sequencing.

It has to be noticed that the subset analysis on some low-abundant cell

445 subpopulations, such as decidual T cells, DC cells and macrophages, remains

446 insufficient. Therefore, it warrants further investigation on the subset

447 characterization of these cells by specifically improved enrichment strategy,

448 which will provide a more comprehensive understanding of the complicated

449 cell-cell communication network at the feto-maternal interface. In addition, the

450 analysis of endometrial cell atlas in the pre-pregnant stage of RSA patients will

451 be helpful to find prospective intervention targets and improve their pregnancy

452 success.

453

454 In general, our study comprehensively illustrates the compromised

455 immune response in periphery and feto-maternal interface of RSA patients.

456 The findings generate a data-driven hypothesis about immune-related 457 pathogenesis for recurrent miscarriage, and provide new insight into the 458 strategies for diagnosis and intervention of the diseases. 
460

461

462

464

465

466

467

468

469

470

471

472

473

474

475

476 477 before the termination of pregnancy.

\section{MATERIALS AND METHODS}

\section{Sample Collection and Ethic permission}

Clinical samples of anti-coagulant peripheral blood and decidual tissues from normal pregnancy $(\mathrm{N}=10)$ or recurrent spontaneous abortions $(\mathrm{RSA})(\mathrm{N}=14)$ at gestational week 6-8 were obtained upon therapeutic termination of pregnancy at Peking University Third Hospital. The decidual tissues were immersed in iced RPMI-1640 medium and the blood samples were kept on ice. All samples were subjected to cell isolation or fixation within 1 hour following the surgery. The collection of human samples was permitted by the Local Ethical Committees in Peking University Third Hospital.

RSA was defined according to the criteria of Practice Committee of the American Society for Reproductive Medicine. In brief, these patients had history of two or more failed pregnancies with unknown cause(Committee and Society, 2013). Women who manifest endocrine disorder, fetal chromosomal or congenital abnormalities, uterine anatomical disorders, renal disease, or pregnancies conceived by fertility treatment were excluded from this study. All the enrolled patients had arrested fetal development for less than one week

\section{Cell isolation and purification}

480 Freshly collected human decidual tissues were trimmed into $1 \mathrm{~mm}$ piece by

481 GentelMACS Dissociator (Miltenyi Biotec, 130-093-235, Germany) and 
482

483

484 485

486

487

488 489

490

491

492

494

495

496

497

498

499

500

501

502

503

digested twice for 30 minutes each at $37^{\circ} \mathrm{C}$ with $1.0 \mathrm{mg} / \mathrm{ml}$ type IV collagenase (Gibco, 9001121, Grand Island, NY, USA) and 10U/ml type I DNase (Sigma, DN25, St Louis, MO, USA). The cell suspensions were filtered through 60 mesh and 200 mesh sieves, and were collected by centrifuging at 1000rpm for $10 \mathrm{~min}$. The resuspended cells were subjected to lymphocyte enrichment using Ficoll-Paque Plus (17-1440-02, GE Healthcare). The decidual leukocytes were further purified by fluorescent activated cell sorting with 7-AAD (420404, Bioledgend) and FITC-labeled anti-CD45 antibody (11-0459-42, eBioscience).

Freshly collected peripheral blood samples were diluted to 1:1 with PBS. Which Peripheral blood after dilution were subjected to lymphocyte enrichment using Ficoll-Paque Plus (17-1440-02, GE Healthcare). The peripheral blood leukocytes were further purified by fluorescent activated cell sorting with 7-AAD (420404, Bioledgend) and FITC-labeled anti-CD45 antibody (11-0459-42, eBioscience).

The freshly purified decidual or peripheral leukocytes were immediately subjected to single cell RNA sequencing as described below.

\section{Generation of single-cell library and transcriptome sequencing}

The purified leukocytes from three pairs of normal and RSA cases were separately loaded on Chromium Single Cell Controller (10x Genomics) using the Chromium Single-cell 3' kit v2 to capture 5000 to 8000 cells per sample. 
504 Libraries were sequenced on an Illumina NovaSeq 6000 (Illumina, San Diego)

505

506 (sample barcode), and 98 bp for read 2 (actual RNA read). Reads were first 507

521 downstream integration, and ran PCA on each individual sample in the

522 combined object. Since our data include RSA patients and normal pregnant 523 women, we chose one of RSA and normal pregnant women data as the 524 reference, and then used the function FindlntegrationAnchors provided by 525 Seurat to integrate the samples of 6 individuals. Downstream analyses, 
526

527

528 543 1:200), or CD103 (350227, Biolegend, 1;200). Binding of the antibody was

544 visualized using FITC-conjugated or TRITC-conjugated secondary antibody 545 (ZSGB-BIO, ZF-0311, ZF-0313, 1:100), and cell nuclei were stained with 4, 546 6-diamidino-2-phenylindole (DAPI; Sigma, 28718-90-3, St Louis, MO, USA). 547 The results were recorded using Zeiss LSM780 confocal system (Zeiss,

including normalization, shared nearest neighbor graph-based clustering, differential expression analysis, and visualization, were performed using the standard workflow provided by Seurat (version 3.0.3). Developmental trajectories were inferred with the Monocle2 (version 2.12.0),. We used the function FindVariableGenes of Seurat to find the variant genes of dNK cells, and then performed trajectory analysis based on the standard workflow provided by Monocle2. We performed differential gene expression analysis of all decidual cell types using Wilcoxon rank sum test inbuilt in Seurat package. differentially expressed genes for dNK clusters were KEGG analysis using $R$ package clusterProfiler(version 3.12.0)(Yu, 2015).

\section{Immunostaining analysis}

Human decidual tissues were briefly fixed in 4\% PFA and embedded in O.C.T. compound (Sakura Finetek, Torrance, CA, USA). The frozen sections at $10 \mathrm{um}$ were further fixed in $4 \%$ PFA and treated with $0.1 \%$ triton, and subjected to the incubation with specific antibodies against NCAM1/CD56 (Abcam, ab75813, 1:300), CK7 (Abcam, ab181598, 1:10000), CD39 (proteintech, 14211-1-AP, 
548 Oberkochen, BW, Germany) and processed with ZEN 2012 software (Zeiss).

549 Immunohistochemical staining for Cytokeratin (CK) in decidua was performed

550 by using antibody against CK7 (ab181598, Abcam,1:10000) and

551 HRP-conjugated second antibody (ZSGB-BIO, PV-6001) followed by recovery

552 of substrate DAB (ZSGB-BIO, ZLI-9019). The imagines were recorded on a

553 light microscope with CCD (DP72, Olympus, Japan).

554

\section{Flow cytometry Assay}

556 Flow cytometry assay for dNK cells was carried out in CytoFLEX (Beckman

557 Coulter, Inc.) using the following antibodies: PE-labeled anti-CD56(362508,

558 Bioledgend), Percp-cy5.5-labeled anti-CD3(300328, Bioledgend), APC-labeled

559 ani-CD39(328209, Bioledgend), APC-cy7-labeled anti-CD103(350227,

560 Bioledgend), FITC-labeled anti-CD16(302206, Bioledgend), PE-labeled

561 anti-CD27(356405, Bioledgend), Pacific blue-labeled anti CD11b(301316,

562 Bioledgend) according to the manufacturer's instructions. Data was analyzed

563 using CytExpert (Beckman Coulter, Inc.) .

564

565

Statistical analysis

566 Comparison of cell proportion between normal and RSA pregnancies were

567 statistically analyzed with GraphPad Prism version 7.00 (GraphPad Software,

568 San Diego, CA, USA). Data were shown as Mean \pm S.E.M. and comparison

569 was carried out by unpaired t-test. The $P$ values of less than 0.05 were 
570

571

572 Seurat.
574

575

576

577

578

579 number

$$
\text { HRA000237 }
$$

that

are

publicly

accessible

580 at http://bigd.big.ac.cn/gsa-human.

\section{ACKNOWLEDGEMENTS}

583 We are grateful to Prof. Bin Cao at Xiamen University and Mr. Zhenghui Zhao

584 at IOZ for their critical comments to this study. The technical support from

585 Shiwen Li, Xia Yang and Qing Meng in the experiments of Confocal analysis

586 and FACS was appreciated. We acknowledge all the enrolled patients for their

587 contribution to the study.

588 This study was supported by grants from the National Key Research and

589 Development Program of China (2018YFC10041002, 2017YFC1001404, 590 2016YFC1000401, 2016YFC1000200), and the National Natural Science 591 Foundation (81730040, 81490740). 
592

593

594

595

596

597

598

599

600

601

602

603

604

605

606

607

608

609

610

611

612

\section{AUTHOR CONTRIBUTIONS}

F.W. performed cell isolation, flow cytometry and scRNA sequencing data analysis, and drafted manuscript. W.J. carried out immunostaining and flow cytometry, and participated in data analysis. M.F. was in charge of patient enrollment, sample collection and clinical data checking. Y.L. participated in scRNA sequencing data analysis and manuscript revision. Z.L. helped in immunostaining and flow cytometry. Y.M., X.S. and Y.L. were involved in data analysis and manuscript drafting. Y.L.W., Q.T. and R.L. designed and supervised the study, interpreted the data and revised the manuscript.

\section{DECLARATION OF INTERESTS}

The authors declare no competing interests.

\section{REFERENCES}

Ander, S.E., Diamond, M.S., and Coyne, C.B. (2019). Immune responses at the maternal-fetal interface. Sci. Immunol.

Arck, P.C., and Hecher, K. (2013). Fetomaternal immune cross-talk and its consequences for maternal and offspring's health. Nat. Med.

Baratin, M., Roetynck, S., Lépolard, C., Falk, C., Sawadogo, S., Uematsu, S., Akira, S., Ryffel, B., Tiraby, J.G., Alexopoulou, L., et al. (2005). Natural killer cell and macrophage cooperation in MyD88-dependent innate responses to Plasmodium falciparum. Proc. Natl. Acad. Sci. U. S. 
614 Bertrand, L., and Lehuen, A. (2019). MAIT cells in metabolic diseases. Mol. Metab.

615 Bulmer, J.N., Morrison, L., Longfellow, M., Ritson, A., and Pace, D. (1991). Granulated

616 lymphocytes in human endometrium: Histochemical and immunohistochemical studies. Hum.

617 Reprod.

618 Butler, A., Hoffman, P., Smibert, P., Papalexi, E., and Satija, R. (2018). Integrating single-cell

619 transcriptomic data across different conditions, technologies, and species. Nat. Biotechnol.

620 Carlino, C., Stabile, H., Morrone, S., Bulla, R., Soriani, A., Agostinis, C., Bossi, F., Mocci, C.,

621 Sarazani, F., Tedesco, F., et al. (2008). Recruitment of circulating NK cells through decidual

622 tissues: A possible mechanism controlling NK cell accumulation in the uterus during early

623 pregnancy. Blood.

624 Committee, P., and Society, A. (2013). Definitions of infertility and recurrent pregnancy loss: A

625 committee opinion. Fertil. Steril. 99, 63.

626 Dalbeth, N., Gundle, R., Davies, R.J.O., Lee, Y.C.G., McMichael, A.J., and Callan, M.F.C.

627 (2004). CD56 bright NK Cells Are Enriched at Inflammatory Sites and Can Engage with

628 Monocytes in a Reciprocal Program of Activation . J. Immunol.

629 Deshmukh, H., and Way, S.S. (2019). Immunological Basis for Recurrent Fetal Loss and

630 Pregnancy Complications. Annu. Rev. Pathol. Mech. Dis.

631 Du, M.R., Wang, S.C., and Li, D.J. (2014). The integrative roles of chemokines at the

632 maternal-fetal interface in early pregnancy. Cell. Mol. Immunol. 
633 Ebina, Y., Nishino, Y., Deguchi, M., Maesawa, Y., Nakashima, Y., and Yamada, H. (2017).

634 Natural killer cell activity in women with recurrent miscarriage: Etiology and pregnancy

635 outcome. J. Reprod. Immunol.

636 Erlebacher, A. (2013). Immunology of the Maternal-Fetal Interface. Annu. Rev. Immunol.

637 Fisher, S.J. (2015). Why is placentation abnormal in preeclampsia? Am. J. Obstet. Gynecol.

638213, S115-S122.

639 Fridman, W.H., Pagès, F., Sauts-Fridman, C., and Galon, J. (2012). The immune contexture in

640 human tumours: Impact on clinical outcome. Nat. Rev. Cancer.

641 Fu, B., Wang, F., Sun, R., Ling, B., Tian, Z., and Wei, H. (2011). CD11b and CD27 reflect

642 distinct population and functional specialization in human natural killer cells. Immunology.

643 Fu, B., Li, X., Sun, R., Tong, X., Ling, B., Tian, Z., and Wei, H. (2013). Natural killer cells

644 promote immune tolerance by regulating inflammatory $\mathrm{TH} 17$ cells at the human maternal-fetal

645 interface. Proc. Natl. Acad. Sci. U. S. A.

646 Gamliel, M., Goldman-Wohl, D., Isaacson, B., Gur, C., Stein, N., Yamin, R., Berger, M.,

647 Grunewald, M., Keshet, E., Rais, Y., et al. (2018a). Trained Memory of Human Uterine NK

648 Cells Enhances Their Function in Subsequent Pregnancies. Immunity 48, 951-962.e5.

649 Gamliel, M., Goldman-Wohl, D., Isaacson, B., Gur, C., Stein, N., Yamin, R., Berger, M.,

650 Grunewald, M., Keshet, E., Rais, Y., et al. (2018b). Trained Memory of Human Uterine NK

651 Cells Enhances Their Function in Subsequent Pregnancies. Immunity.

652 Gentek, R., Molawi, K., and Sieweke, M.H. (2014). Tissue macrophage identity and 
653

654 Godfrey, D.I., Koay, H.F., McCluskey, J., and Gherardin, N.A. (2019). The biology and

655 functional importance of MAIT cells. Nat. Immunol.

656 Haghverdi, L., Lun, A.T.L., Morgan, M.D., and Marioni, J.C. (2018). Batch effects in single-cell RNA-sequencing data are corrected by matching mutual nearest neighbors. Nat. Biotechnol.

Hanna, J., Wald, O., Goldman-Wohl, D., Prus, D., Markel, G., Gazit, R., Katz, G.,

659 Haimov-Kochman, R., Fujii, N., Yagel, S., et al. (2003). CXCL12 expression by invasive

660 trophoblasts induces the specific migration of CD16- human natural killer cells. Blood.

661 Hanna, J., Goldman-Wohl, D., Hamani, Y., Avraham, I., Greenfield, C., Natanson-Yaron, S.,

662 Prus, D., Cohen-Daniel, L., Arnon, T.I., Manaster, I., et al. (2006). Decidual NK cells regulate

663 key developmental processes at the human fetal-maternal interface. Nat. Med.

664 Huhn, O., Ivarsson, M.A., Gardner, L., Hollinshead, M., Stinchcombe, J.C., Chen, P., Shreeve,

665 N., Chazara, O., Farrell, L.E., Theorell, J., et al. (2020). Distinctive phenotypes and functions

666 of innate lymphoid cells in human decidua during early pregnancy. Nat. Commun.

667 Kheshtchin, N., Gharagozloo, M., Andalib, A., Ghahiri, A., Maracy, M.R., and Rezaei, A. (2010).

668 The expression of Th1- and Th2-related chemokine receptors in women with recurrent

669 miscarriage: The impact of lymphocyte immunotherapy. Am. J. Reprod. Immunol.

670 King, A., Balendran, N., Wooding, P., and Loke, Y.W. (1991). Cd3- Leukocytes Present in the

671 Human Uterus During Early Placentation: Phenotypic and Morphologic Characterization of the

672 Cd56++ Population. Dev. Immunol. 
673 King, K., Smith, S., Chapman, M., and Sacks, G. (2010). Detailed analysis of peripheral blood

674 natural killer (NK) cells in women with recurrent miscarriage. Hum. Reprod.

675 Klentzeris, L.D., Bulmer, J.N., Warren, M.A., Morrison, L., Li, T.C., and Cooke, I.D. (1994).

676 Lymphoid tissue in the endometrium of women with unexplained infertility: Morphometric and

677 immunohistochemical aspects. Hum. Reprod.

678 Kuon, R.J., Vomstein, K., Weber, M., Müller, F., Seitz, C., Wallwiener, S., Strowitzki, T.,

679 Schleussner, E., Markert, U.R., Daniel, V., et al. (2017). The "killer cell story" in recurrent

680 miscarriage: Association between activated peripheral lymphocytes and uterine natural killer

681 cells. J. Reprod. Immunol.

682 Lachapelle, M.H., Miron, P., Hemmings, R., and Roy, D.C. (1996). Endometrial T, B, and NK

683 cells in patients with recurrent spontaneous abortion. Altered profile and pregnancy outcome. J.

684 Immunol.

685 Ma, L., Li, G., Cao, G., Zhu, Y., Du, M.R., Zhao, Y., Wang, H., Liu, Y., Yang, Y., Li, Y.X., et al.

686 (2017). DNK cells facilitate the interaction between trophoblastic and endothelial cells via

687 VEGF-C and HGF. Immunol. Cell Biol.

688 Manaster, I., Mizrahi, S., Goldman-Wohl, D., Sela, H.Y., Stern-Ginossar, N., Lankry, D., Gruda,

689 R., Hurwitz, A., Bdolah, Y., Haimov-Kochman, R., et al. (2008). Endometrial NK Cells Are

690 Special Immature Cells That Await Pregnancy. J. Immunol.

691 Nancy, P., Tagliani, E., Tay, C.S., Asp, P., Levy, D.E., and Erlebacher, A. (2012). Chemokine

692 gene silencing in decidual stromal cells limits T cell access to the maternal-fetal interface.

693 Science7. 
694 Parham, P., and Moffett, A. (2013). Variable NK cell receptors and their MHC class i ligands in

695 immunity, reproduction and human evolution. Nat. Rev. Immunol.

696 PrabhuDas, M., Bonney, E., Caron, K., Dey, S., Erlebacher, A., Fazleabas, A., Fisher, S.,

697 Golos, T., Matzuk, M., McCune, J.M., et al. (2015). Immune mechanisms at the maternal-fetal

698 interface: Perspectives and challenges. Nat. Immunol.

699 Seshadri, S., and Sunkara, S.K. (2014). Natural killer cells in female infertility and recurrent

700 miscarriage: A systematic review and meta-analysis. Hum. Reprod. Update.

701 Sotnikova, N., Voronin, D., Antsiferova, Y., and Bukina, E. (2014). Interaction of decidual

702 CD56+ NK with trophoblast cells during normal pregnancy and recurrent spontaneous abortion

703 at early term of Gestation. Scand. J. Immunol.

704 Stuart, T., Butler, A., Hoffman, P., Hafemeister, C., Papalexi, E., Mauck, W.M., Hao, Y.,

705 Stoeckius, M., Smibert, P., and Satija, R. (2019). Comprehensive Integration of Single-Cell

706 Data. Cell.

707 Suryawanshi, H., Morozov, P., Straus, A., Sahasrabudhe, N., Max, K.E.A., Garzia, A., Kustagi,

708 M., Tuschl, T., and Williams, Z. (2018). A single-cell survey of the human first-trimester

709 placenta and decidua. Sci. Adv.

710 Vacca, P., Vitale, C., Montaldo, E., Conte, R., Cantoni, C., Fulcheri, E., Darretta, V., Moretta,

711 L., and Mingari, M.C. (2011). CD34+ hematopoietic precursors are present in human decidua

712 and differentiate into natural killer cells upon interaction with stromal cells. Proc. Natl. Acad.

713 Sci. U. S. A.

714 Varol, C., Mildner, A., and Jung, S. (2015). Macrophages: Development and Tissue 
716 Vento-Tormo, R., Efremova, M., Botting, R.A., Turco, M.Y., Vento-Tormo, M., Meyer, K.B.,

717 Park, J.E., Stephenson, E., Polański, K., Goncalves, A., et al. (2018). Single-cell

718 reconstruction of the early maternal-fetal interface in humans. Nature.

719 Wallace, A.E., Host, A.J., Whitley, G.S., and Cartwright, J.E. (2013). Decidual natural killer cell

720 interactions with trophoblasts are impaired in pregnancies at increased risk of preeclampsia.

$721 \quad$ Am. J. Pathol.

722 Wang, F., Zhou, Y., Fu, B., Wu, Y., Zhang, R., Sun, R., Tian, Z., and Wei, H. (2014). Molecular

723 signatures and transcriptional regulatory networks of human immature decidual NK and

724 mature peripheral NK cells. Eur. J. Immunol. 44, 2771-2784.

725 Wang, W.J., Hao, C.F., Yi-Lin, Yin, G.J., Bao, S.H., Qiu, L.H., and Lin, Q. De (2010). Increased

726 prevalence of T helper 17 (Th17) cells in peripheral blood and decidua in unexplained

727 recurrent spontaneous abortion patients. J. Reprod. Immunol.

728 Wang, Y., Song, F., Zhu, J., Zhang, S., Yang, Y., Chen, T., Tang, B., Dong, L., Ding, N., Zhang,

729 Q., et al. (2017). GSA: Genome Sequence Archive*. Genomics, Proteomics Bioinforma. 15,

$730 \quad 14-18$.

731 Wu, X., Jin, L.-P., Yuan, M.-M., Zhu, Y., Wang, M.-Y., and Li, D.-J. (2005). Human

732 First-Trimester Trophoblast Cells Recruit CD56 bright CD16 - NK Cells into Decidua by Way

733 of Expressing and Secreting of CXCL12/Stromal Cell-Derived Factor 1. J. Immunol.

734 Yang, F., Zheng, Q., and Jin, L. (2019). Dynamic Function and Composition Changes of

735 Immune Cells During Normal and Pathological Pregnancy at the Maternal-Fetal Interface. 
737 Yang, H., Qiu, L., Chen, G., Ye, Z., Lü, C., and Lin, Q. (2008). Proportional change of

738 CD4+CD25+ regulatory T cells in decidua and peripheral blood in unexplained recurrent

739 spontaneous abortion patients. Fertil. Steril.

$740 \mathrm{Yu}, \mathrm{G}$. (2015). Statistical analysis and visualization of functional profiles for gene and gene

741 clusters. Bioconductor.Org.

742 Zhang, J., Dunk, C., Croy, A.B., and Lye, S.J. (2016). To serve and to protect: the role of

743 decidual innate immune cells on human pregnancy. Cell Tissue Res.

744 Zhang, Z., Zhao, W., Xiao, J., Bao, Y., He, S., Zhang, G., Li, Y., Zhao, G., Chen, R., Gao, Y., et

745 al. (2020). Database Resources of the National Genomics Data Center in 2020. Nucleic Acids

746 Res.

747 Zheng, G.X.Y., Terry, J.M., Belgrader, P., Ryvkin, P., Bent, Z.W., Wilson, R., Ziraldo, S.B.,

748 Wheeler, T.D., McDermott, G.P., Zhu, J., et al. (2017). Massively parallel digital transcriptional 749 profiling of single cells. Nat. Commun.

750 Zhou, Y., Fu, B., Xu, X., Zhang, J., Tong, X., Wang, Y., Dong, Z., Zhang, X., Shen, N., Zhai, Y.,

751 et al. (2020). PBX1 expression in uterine natural killer cells drives fetal growth. Sci. Transl.

752 Med.

\section{FIGURE LEGENDS}

756 Fig. 1. Landscape of immune cells from peripheral blood and decidual tissues in normal 
757 pregnant women and RSA patients at early pregnancy using single-cell RNA-seq.

758 (A) UMAP plot of scRNA-seq data to show the 22 leukocyte clusters in peripheral blood

759 and decidual tissues at early pregnancy. (B) UMAP plot showing cell populations from

760 peripheral blood and decidual tissue. (C) UMAP visualization of cell clustering in normal

761 and RSA pregnancies. (D) Characteristics of the 22 leukocyte clusters based on

762 canonical marker genes expression for cell type annotation.

763

764 Fig. 2. Characteristics of the peripheral blood leukocyte clusters indicate the maternal 765 immune inflammatory status in RSA patients.

766 (A) Heatmap of the top5 genes expressed in peripheral blood $T$ cell subsets. $x$-axis

767 represents $\mathrm{T}$ cell clusters; y-axis represents genes. (B) Box plots depicting cell 768 composition of peripheral blood T cells in healthy control and RSA patients. (C) Violin plots 769 of selected markers identified by differential expression analysis comparing the MAIT 770 subset between RSA and CTRL patients. (D) UMAP visualization of the log-transformed, 771 normalized IFNG expression showing the relatively stronger cytotoxicity of CD8 T effector 772 in RSA pregnancy. high expression is showed in red, low expression is illustrated in grey.

773 (E) Box plots depicting cell number ratio of peripheral NK cell subsets in peripheral blood

774 of normal and RSA pregnancies. (F) Violin plots of indicated genes identified by differential 775 expression analysis of NK dim subset between RSA and CTRL patients. Differential 776 expression analyses in Fig. 2 were performed using the non-parametric two-sided 777 Wilcoxon rank sum test in Seurat. 
779 Fig. 3. Characteristics of decidual leukocyte subpopulations demonstrated an immune

780 activation status at the maternal-fetal interface of RSA pregnancy.

781 (A) Cell proportion of T, dM and dNK cell subsets in decidual tissues of normal and RSA

782 pregnancies. (B) and (C) Analysis of the ligand-receptor interactions among the leukocyte

783 subpopulations between RSA and normal pregnancies in decidua. C: CTRL, R: RSA. (D)

784 Volcano plot showing globally differential genes in decidual macrophages between normal

785 and RSA pregnancies. (E) Expression of anti-inflammation and inflammation -associated

786 cytokines in decidual macrophage subpopulation in normal and RSA pregnancies.

787 Differential expression analyses in $\mathrm{D}$ were performed using the non-parametric two-sided

788 Wilcoxon rank sum test in Seurat. Panels

789

790 Fig. 4. Molecular aspects and developmental trajectories of human dNK subtypes

791 (A) Heatmap showing relative expression (z-score) of 20 marker genes defining the

792 subpopulations of dNK cells. (B) Functional KEGG enrichment analysis illustrating the

793 functional signature of the dNK subpopulations. (C) Expression of KIR molecules

794 mediating in the indicated dNK subpopulations. (D) Flow cytometry analysis showing the

795 dNKc cells do not express CD27 and CD11b, further demonstrating their

796 low-differentiation status. (E) Monocle2 analysis showing the developmental trajectories

797 of dNK cells. (F) The expression of classical transcription factors in dNK subpopulations

798 indicating their different developmental status.

800 Fig. 5. Immunofluorescence to show the spatial distribution of dNK cells at the 
801

802

803

806

807

810

Fig. S1. Gating strategy and quality control for droplet single-cell RNA-seq.

821 (A) Flow Sorting of living leukocytes from peripheral blood and decidual tissue using CD45

822 antibody and 7AAD. (B) Workflow to depict the dissociation and sorting of CD45+

\section{feto-maternal interface}

(A) Immunohistochemistry for cytokeratin 7 (CK7) was used to locate trophoblast and uterine gland epithelium in the sections. (B) and (C) Immunofluorescence staining show the spatial distribution of dNKc (CD39+) and dNKd (CD103+) cells at the feto-maternal interface at early pregnancy. Scale bar in panel B and C from left to right are 100, 25, 10 $\mu m$. (D) The spatial distribution of dNKe in decidual tissues from normal and RSA pregnancies by Immunofluorescence staining. Scale bar of panel $D, 25 \mu \mathrm{m}$. The arrow marks the target cell we zoomed in Fig. 5 B, C and D. The dotted line in panel D indicates the position of blood vessels

Fig. 6. Alterations of dNK subsets in decidua of RSA pregnancy

(A) Flow cytometry to sort dNKc (CD39+), dNKd (CD103+) and dNKe(CD16+) cells in human decidual tissues. (B) Cell population of $d N K c$ and $d N K d$ in decidua tissues from normal pregnancy $(n=5)$ and RSA $(n=5)$. Lower panel, Statistical results showing cell proportions of dNKe in decidua tissues from normal pregnancy $(n=4)$ and RSA $(n=11)$. (C) Violin plots revealing differential expression of indicated genes in dNKc, dNKd and dNKe subsets from RSA and CTRL patients. Significance was analyzed using unpaired t-tests assuming no consistent s.d.. 
leukocytes from peripheral blood and decidual to generate scRNA transcriptome profile.

824 (C)Total number of cells that met the requirement of quality control and subjected to

825 droplet scRNA-seq. (D) Quality evaluation on sequencing data including nFeature_RNA

826 and nCount_RNA in peripheral blood and decidua. E, Every cluster distribution of each

827 cell subpopulation in the 6 samples.

828

829 Fig. S2. The MNN algorithm to confirm the appropriate elimination of the batch effect of 830 the data.

831 (A) Data from the 6 samples were integrated with MNN and visualized using UMAP. (B)

832 Distribution of leukocytes from peripheral blood and decidual tissues after MNN

833 integration. (C) Mutual nearest neighbors (mnn_1 and mnn_2) of the integrated analysis

834 of decidual and peripheral blood leukocytes from the droplet-based datasets.

835 Distribution of leukocytes from normal pregnancy and RSA after MNN integration.

836

837 Fig. S3. Identification of peripheral blood T cell subpopulations by specific marker genes.

838 (A) UMAP visualization of peripheral blood T cells. (B)-(E), Feature plot of Naïve T (B),

839 MAIT T (C), CD4T memory (D) and CD8T effector (E) cells with specific marker genes.

841 Fig. S4. Top100 genes in dNK cells that change in a continuous manner over pseudotime

842 (A) Heatmap showing the top100 genes in dNK cells that change in a continuous manner 
845 Fig. S5. Globally differential genes in CD16+ NK cell between dNKe from decidua and NK

846 dim from peripheral blood

847 (A) Volcano plot shows that upregulated and downregulated genes between dNKe and

848 NK dim. Differential expression analyses in dNKe were performed using the 849 non-parametric two-sided Wilcoxon rank sum test in Seurat.

850

851 Fig. S6. Identification of decidual macrophage subpopulations

852 (A) UMAP showing two subpopulations of decidual macrophage. (B) UMAP showing 853 macrophage subpopulations in normal pregnancy (grey) and RSA (red). (C)

854 Characterization of the two macrophage subpopulations using marker genes. 
Aig. 1

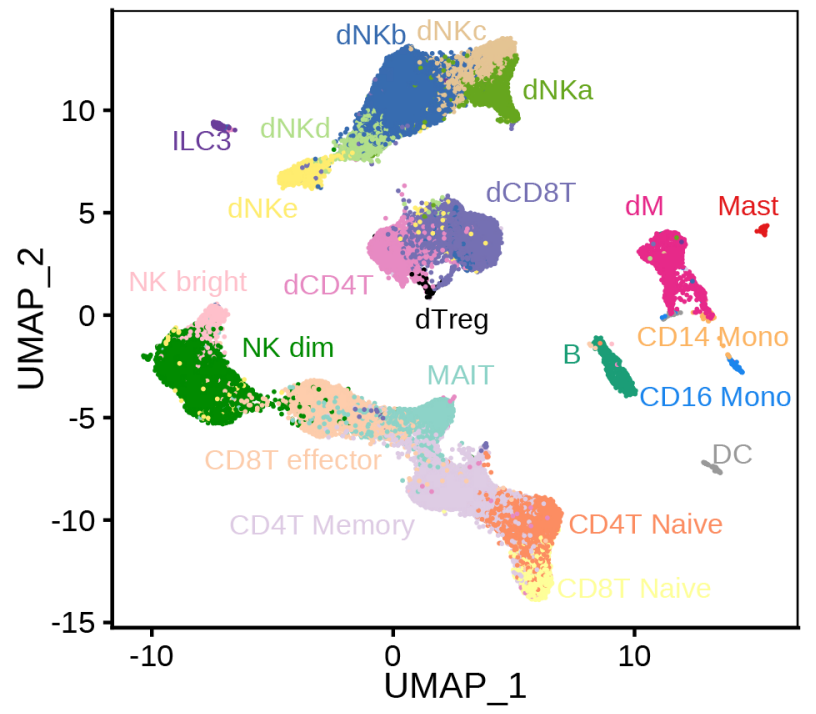

B

C

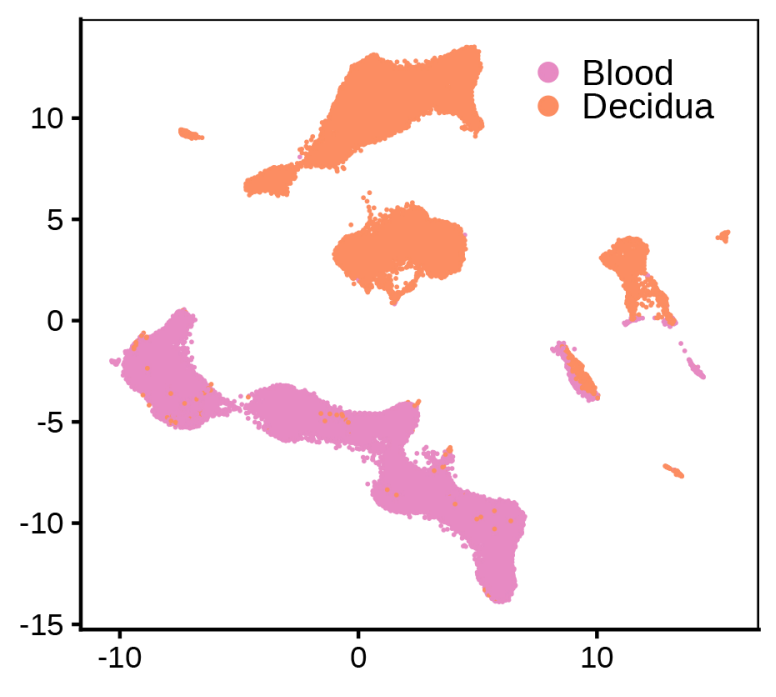

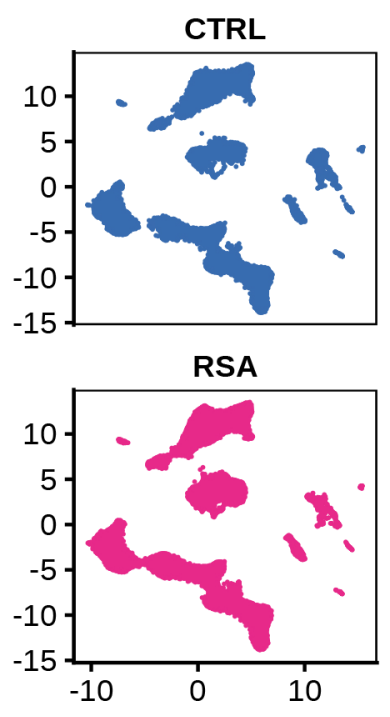

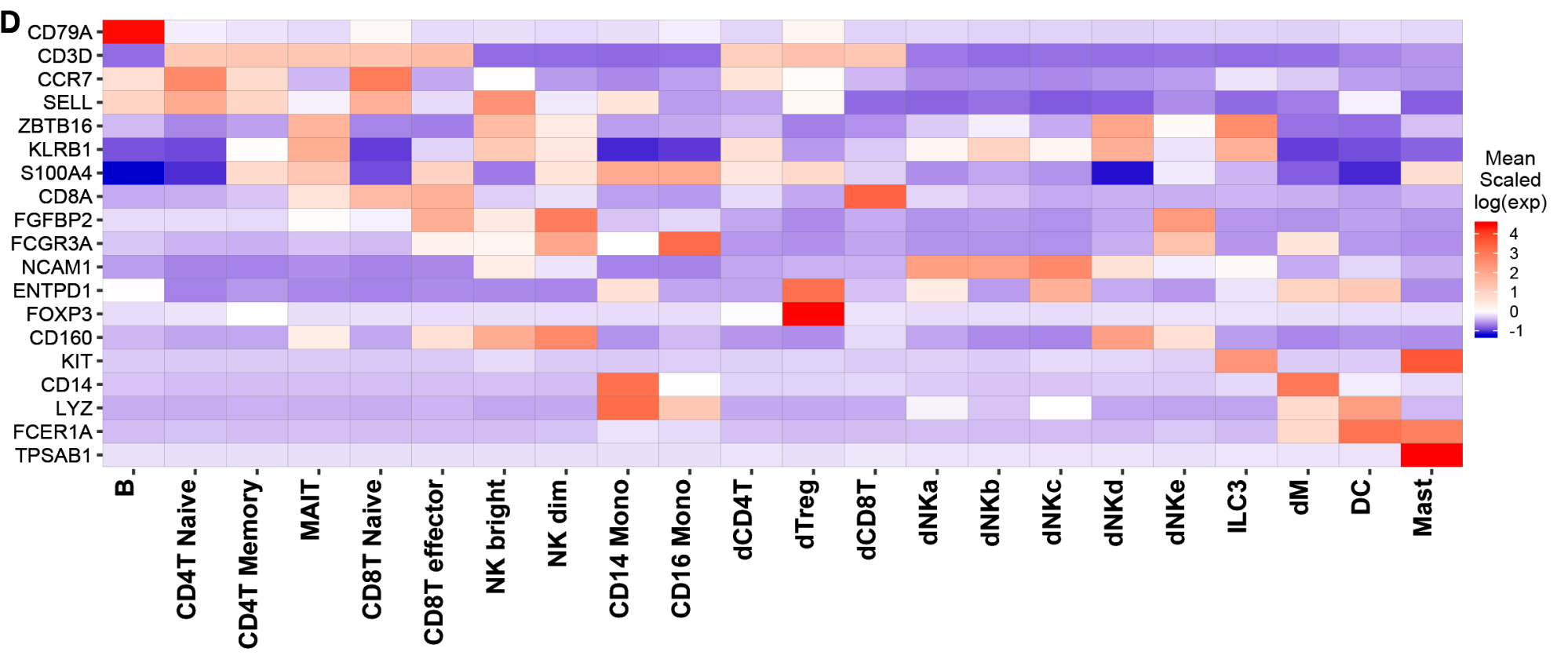


FA. 4
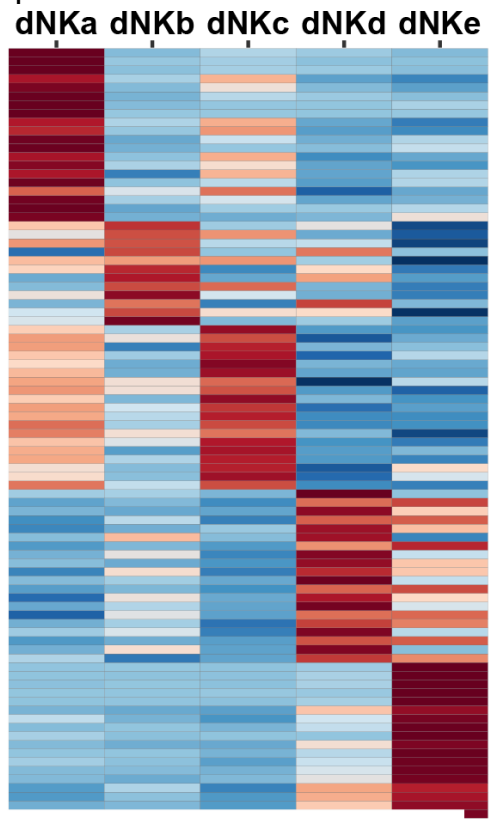

MCM5

STMN1

PCNA

XCL1

XCL2

ZNF683

ENTPD1

PKM

CSF1

ITGAE

CD160

KLRB1

FCGR3A

CXCR4

PLAC8

Mean Scaled log(exp)

C

dNKKa dNKKb dN!Kc dṆd dṆKe $E$

HLA-C

KIR2DL3

HLA-G

LILRB1

HLA-E

D

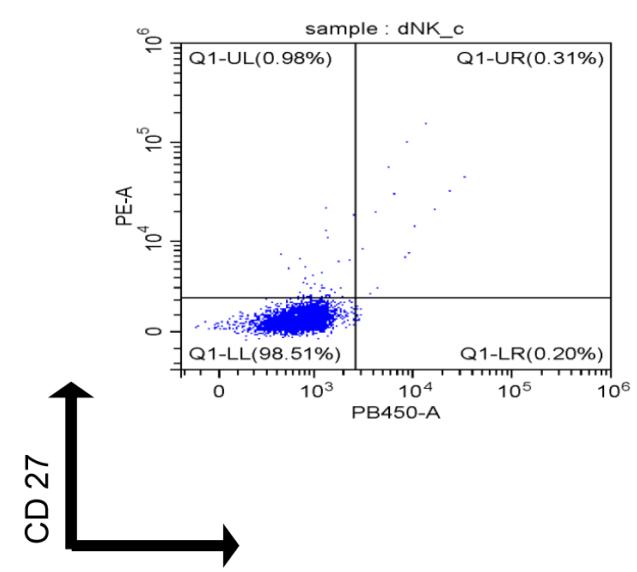

CD11b

B

F $\underset{\frac{\Phi}{d}}{\bar{d}}$ TP53
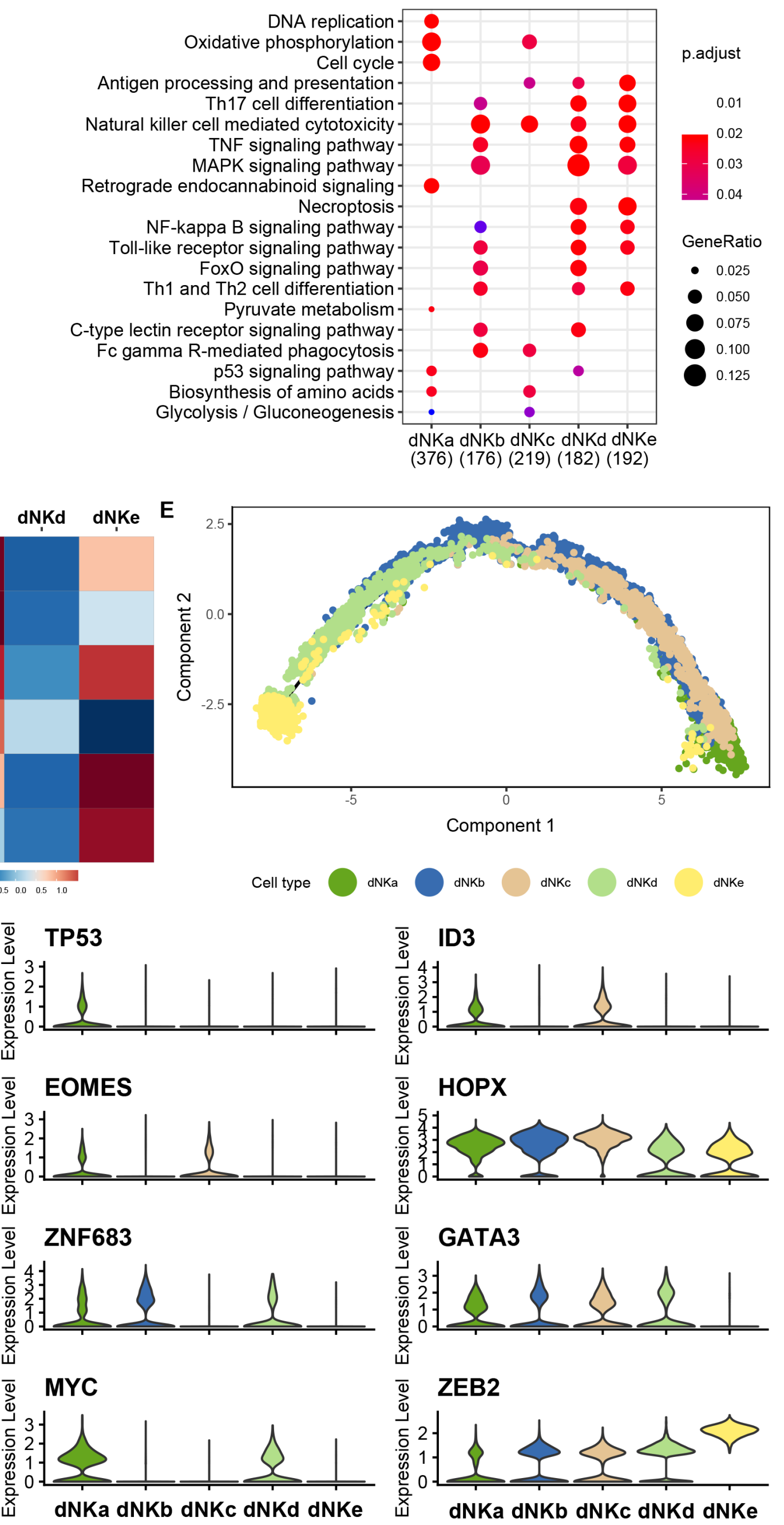
A

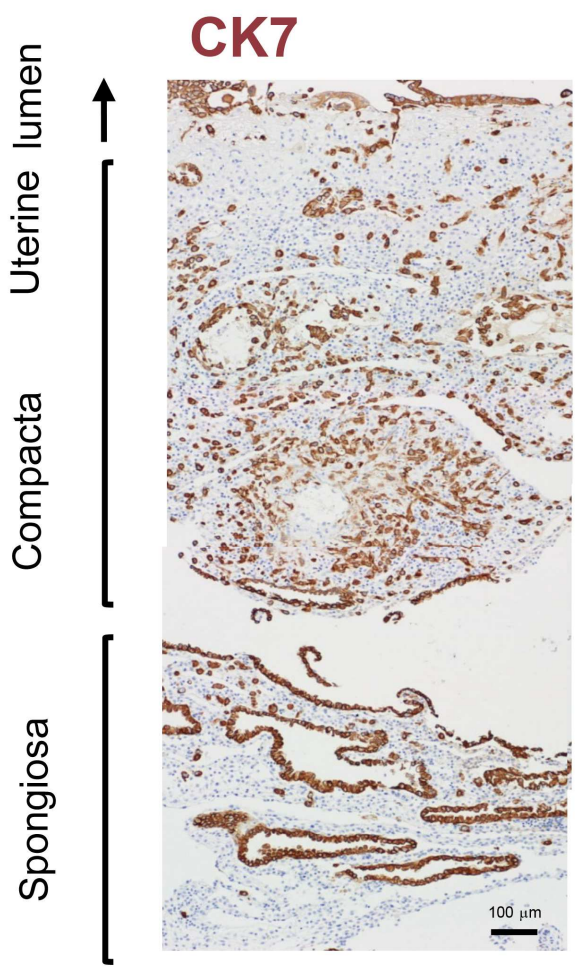

B

\section{CD56 CD103 DAPI}

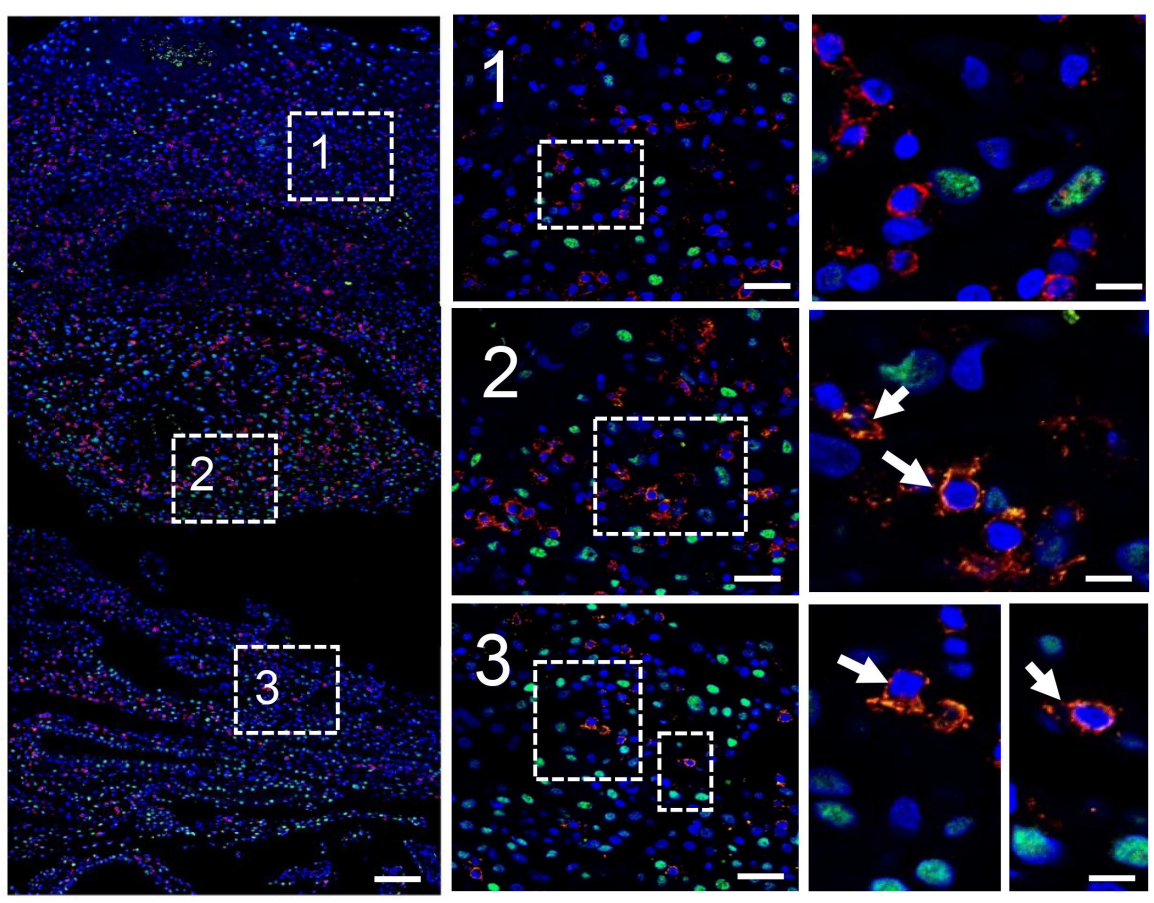

c

\section{CD56 CD39 DAPI}

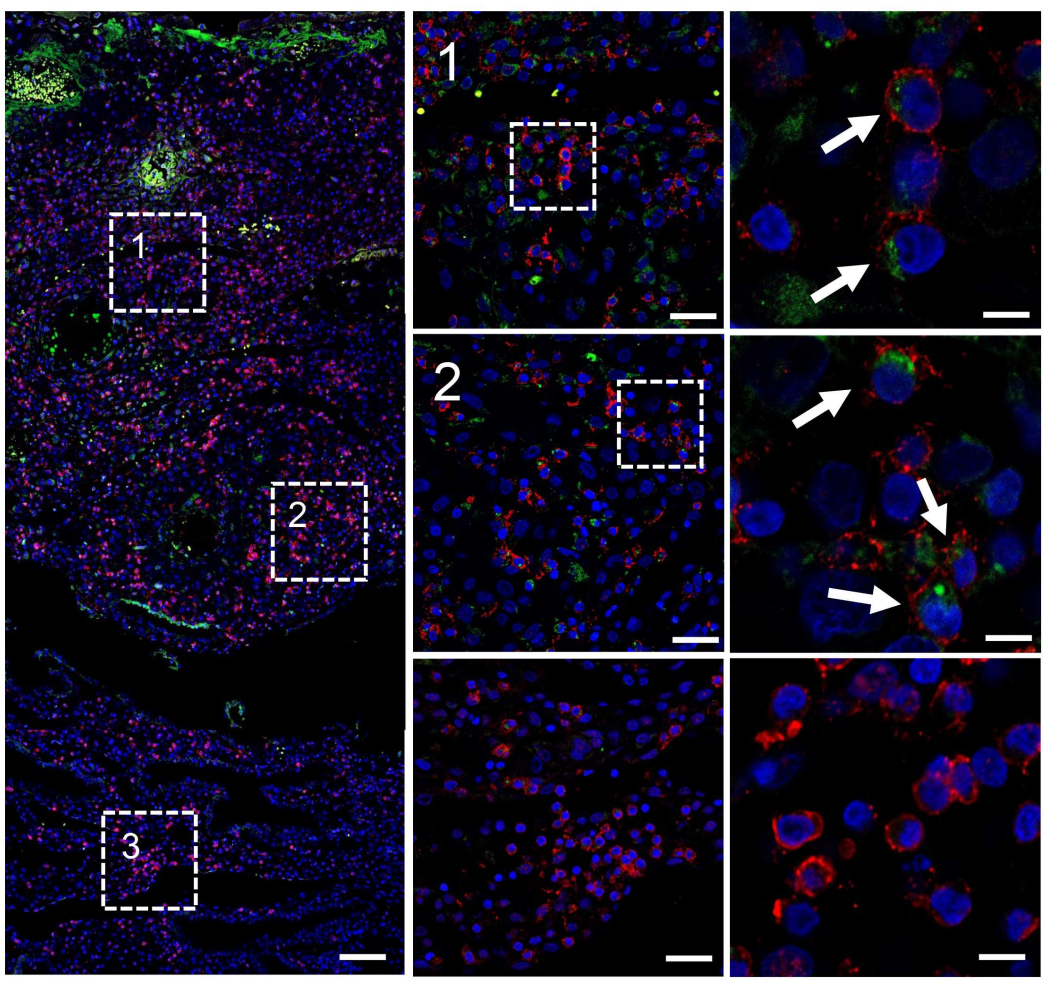

\section{CD16 PLAC8 DAPI}
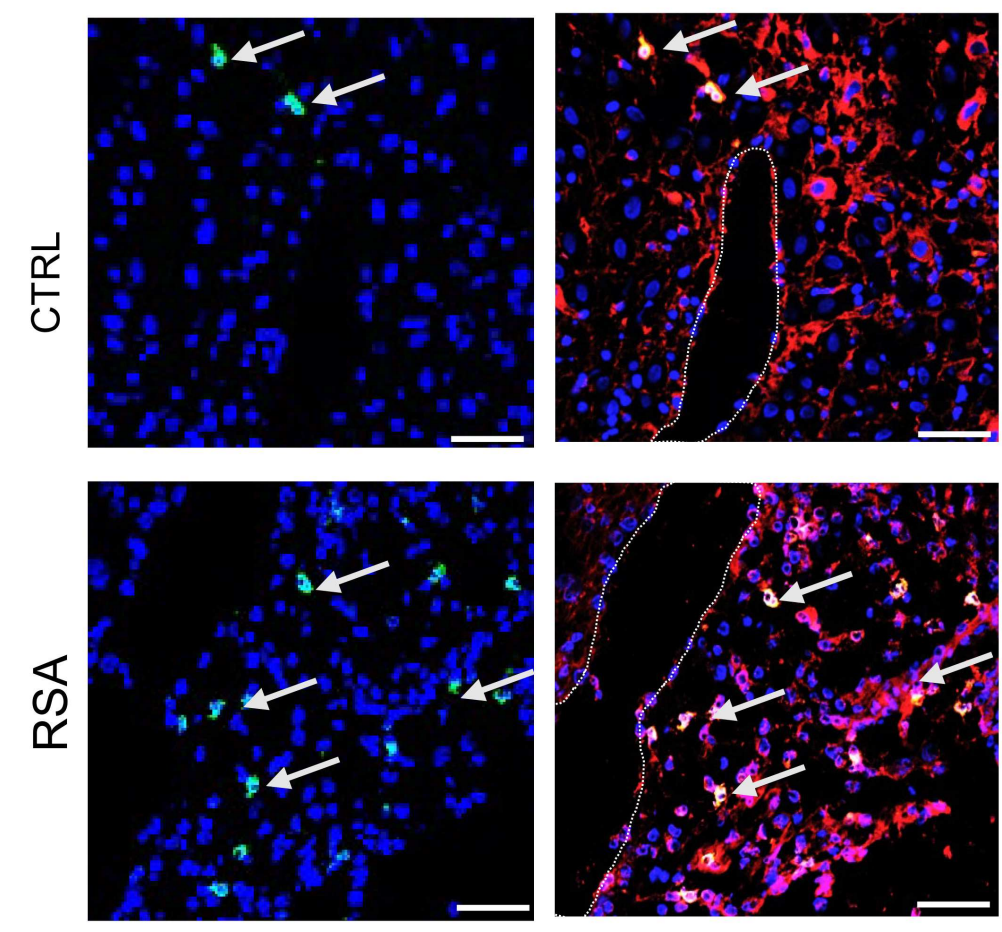
Fig. 6

A

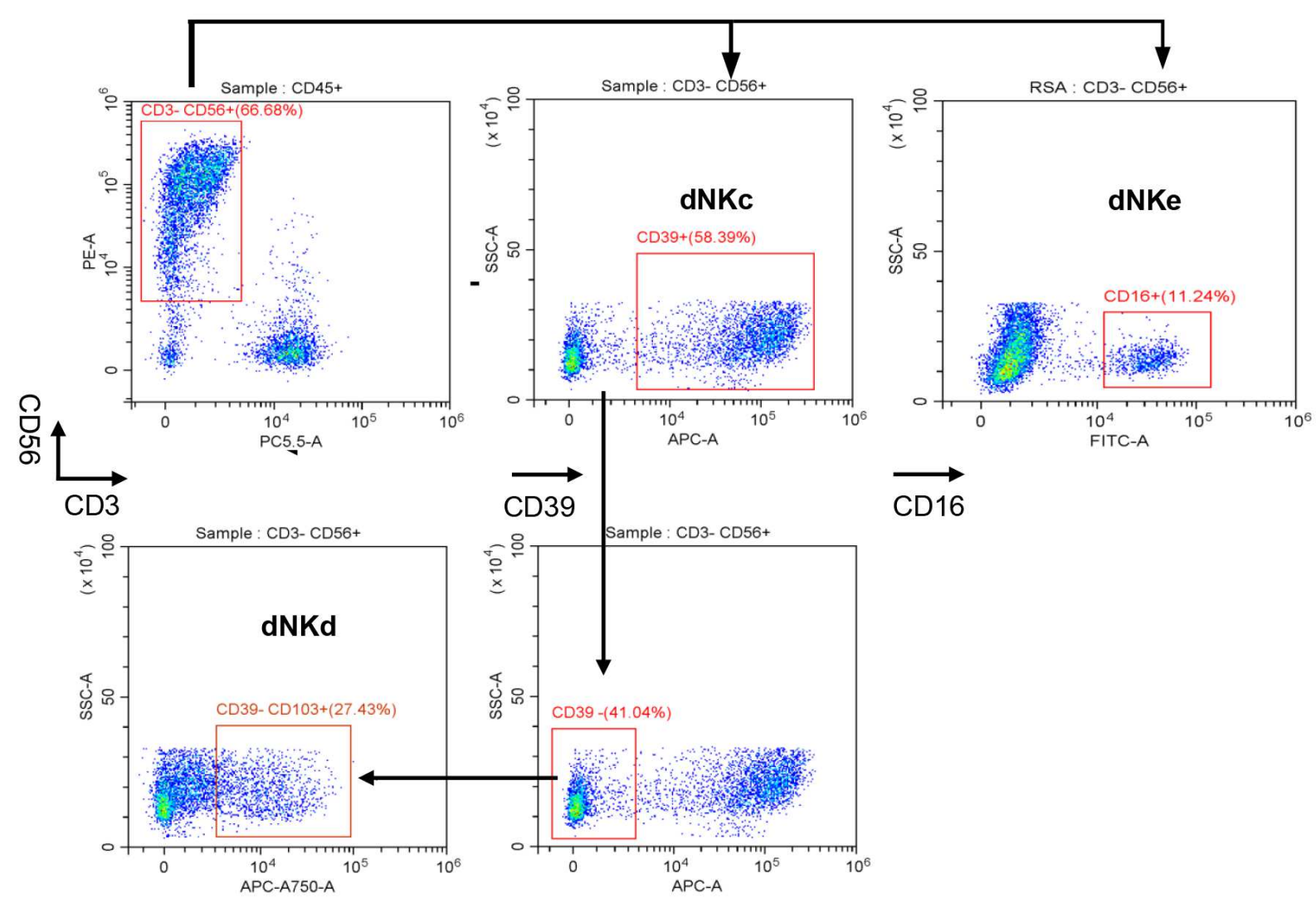

B

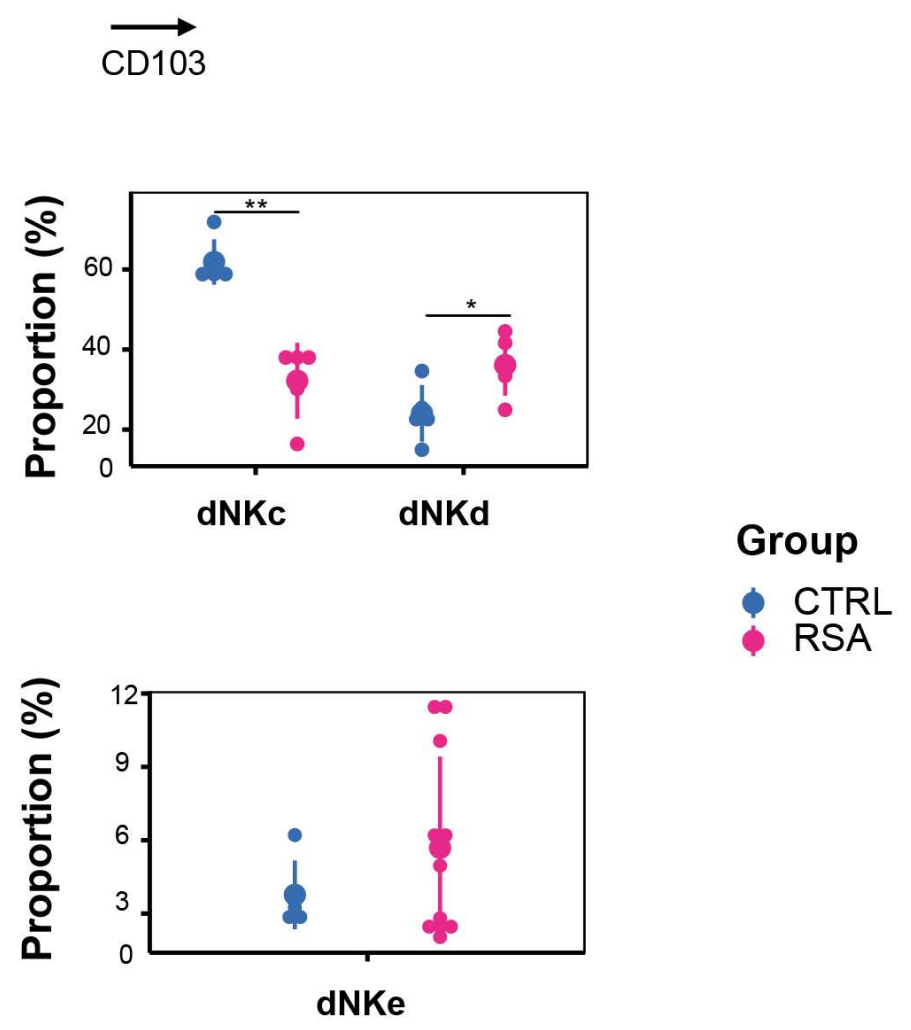

C

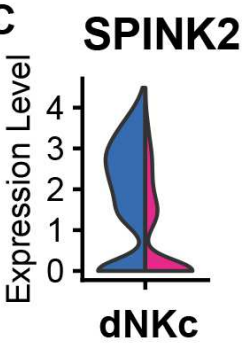

AREG
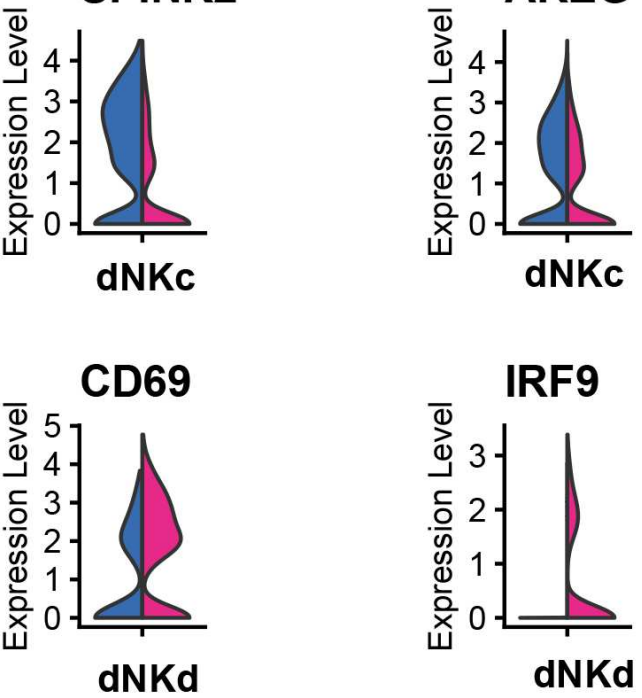

CTRL RSA
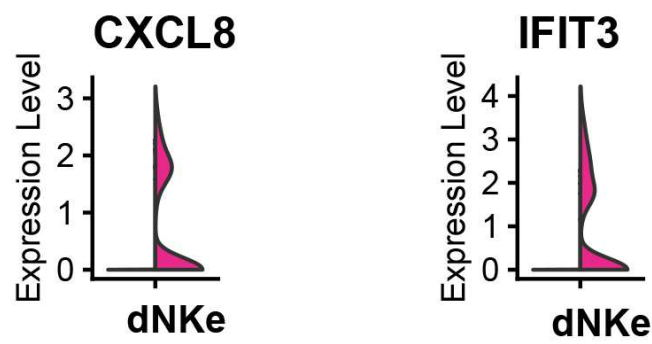